\title{
Benk, Andreas (Hg.) (20 I9). Globales Lernen. Bildung unter dem Leitbild weltweiter Gerechtigkeit. Ostfildern: Matthias Grünewald Verlag. ISBN: 978-3-7867-3200-6. 230 Seiten.
}

\author{
Gudrun Spahn-Skrotzki \\ Universität Kassel (g.spahnsk@uni-kassel.de)
}

\begin{abstract}
„Der Klimawandel ist Realität. Seine negativen Auswirkungen betreffen ohnehin schon Benachteiligte besonders hart. Heutige Entscheidungen beeinflussen das künftige Klima. Von diesen Entscheidungen sind die Lebenschancen nachfolgender Generationen abhängig“ (7).
\end{abstract}

Aus Sicht ganz unterschiedlicher Fächer und Fachdisziplinen greift der von Andreas Benk herausgegebene Band „Globales Lernen“ die drängende Auseinandersetzung mit dem Klimawandel und globalen Gerechtigkeitsfragen auf. Alle Beiträge des Buches wurden als Vorträge einer Ringvorlesung an der Pädagogischen Hochschule Schwäbisch Gmünd gehalten. Gerade die Multiperspektivität des Buches ist seine große Stärke. Es wird deutlich: Klimawandel und globale Auswirkungen unseres Lebensstils weltweit sind dramatisch und fordern unterschiedlichste Fachdisziplinen heraus. So finden sich Beiträge aus Theologie, Philosophie, Ökonomie, Biologie, Kunst, Politikwissenschaft, Ernährungswissenschaft, Genderforschung u. v. a.

Es gibt keine einfachen Lösungen, dieses zeigen die verschiedenen Autor*innen auf, sondern Multiperspektivität und Interdisziplinarität sind notwendig. In dem Sammelband finden sich Auseinandersetzungen mit dem globalisierten Wirtschaftssystem, unserer problematischen Ernährungssituation (z. B. Fleischproduktion in Zusammenhang mit Hunger in der Welt, Klimawandel...), dem Externalisieren von Produktionen in Länder des globalen Südens (Beispiel Kleidung), den ökologischen Auswirkungen von Kunststoffen, der Situation von Frauen und Mädchen weltweit u. v. a.

Forderungen, Konsequenzen und Perspektiven der einzelnen Autor*innen sind z. T. sehr heterogen und unterschiedlich weitreichend. Dieses spiegelt aber auch die gesellschaftliche Diskussion um Klimawandel und Gerechtigkeitsfragen wider.

Es ist sehr erfreulich, dass der Band nicht allein theoretischen und akademischen Diskussionen gewidmet ist, sondern auch jenseits von Fachsprache ein breites Publikum erreichen will und viele Beiträge konkret Veränderung sowohl im Verhalten einzelner als auch im politisch-gesellschaftlichen System erreichen möchten. Die Notwendigkeit zu handeln, zu gesellschaftlich-wirtschaftlicher Veränderung, aber auch zu persönlichen Verhaltensänderungen, wird in vielen Beiträgen deutlich - auch wenn Schwerpunkte in den einzelnen Beiträgen ganz unterschiedlich gesetzt sind.

Ebenfalls positiv zu erwähnen ist, dass in diesem Band Bildung für nachhaltige Entwicklung (BNE) und Globales Lernen nicht mehr in Konkurrenz zu einander gesehen werden, sondern deutlich wird, dass Ausbeutungsstrukturen sowohl gegenüber Menschen als auch gegenüber der Mitwelt sehr häufig zusammenhängen und beides durch den externalisierenden Lebensstil des globalen Nordens bedingt sind: Kosten für den eigenen Wohlstand werden auf andere (vornehmlich Menschen im globalen Süden und die Mitwelt) abgewälzt.

Für die didaktische Aufbereitung der Themenkomplexe Klimawandel, globale Gerechtigkeitsfragen, Auswirkungen unseres Lebensstils liefert der Band viele Anregungen. Lehrende haben die Chance, aus Perspektive anderer Fächer Impulse zu bekommen und diese in Unterricht und Lehre einzubeziehen. Das Buch macht Lust auf fächerübergreifendes und interdisziplinäres Lehren, Forschen und Unterrichten und macht deutlich, dass ein Inhalt wie "globales Lernen" eigentlich auch nur angemessen interdisziplinär und fächerübergreifend thematisiert werden kann. Es wird in vielen Beiträgen aber auch offenkundig, wie nötig eine gründliche Auseinandersetzung mit diesen Inhalten des globalen Lernens in der Schule ist.

Aus religionspädagogischer Sicht ist der Beitrag von Andreas Benk besonders hervorzuheben. Die globalen Aus- 
beutungsstrukturen sind erschütternd und es ist erstaunlich, wie wenig dieses in der Religionspädagogik bislang im Fokus ist. Da ist der Aufsatz von Andreas Benk ein Lichtblick, der die gründliche Auseinandersetzung mit diesen von Theolog*innen und Religionslehrer*innen nun endlich konsequent einfordert. Benk macht darauf aufmerksam, dass globales Lernen keine Zusatzaufgabe, sondern Kernaufgabe des Religionsunterrichts sein muss und er mahnt an, dass die Auseinandersetzung mit globalen Unrechtsstrukturen bislang viel zu wenig im Religionsunterricht thematisiert wird. „Nicht Tod und Jenseitsspekulationen geben in der Bibel Anlass, von Gott zu sprechen, sondern herrschende Unrechtsverhältnisse. Insbesondere die biblische Prophetie entzündet sich an sozialen Missständen. [...] Prophetische Visionen wollen nicht auf ein Jenseits vertrösten, sondern aufrütteln und dazu ermutigen, sich an dem gottgewollten Projekt einer gerechten Gesellschaft zu beteiligen“ (214). So betont er, dass Jesu Reich-Gottes-Botschaft in der Tradition biblischer Theologie steht, „,auf die irdische Verwirklichung der Gerechtigkeit Gottes" zielt und damit einem sozialethischen Anspruch verbunden ist. Aus biblischer Sicht werden "gerechte gesellschaftliche Verhältnisse eingefordert“ (214).

Es ist offenkundig, dass christliche Kirchen diesem Anspruch nur sehr ungenügend entsprochen haben bzw. entsprechen und Benk kritisiert diesbezüglich auch die deutschsprachige Religionspädagogik: „Auch die deutschsprachige Religionspädagogik befasste sich in den vergangenen Jahrzehnten vorzugsweise mit individuellen religiösen Bildungsprozessen und den diese Prozesse prägenden kulturellen Phänomenen“ (216). Hier fordert er ein Umdenken in der Religionspädagogik und betont, dass Religionsunterricht wichtige Impulse zu globalen Gerechtigkeitsfragen setzen kann, stellt aber auch fest, dass Religionslehrkräfte häufig auch nur sehr unzureichend diesbezüglich ausgebildet sind. Damit weist er auf ein Problem in der Ausbildung von Religionslehrer*innen hin. Bislang werden Inhalte wie Nachhaltigkeit, globale Gerechtigkeit, Klimawandel, Verlust von Biodiversität etc. kaum in der Ausbildung von Religionslehrer*innen thematisiert.

Der Aufsatz von Andreas Benk liefert einen sehr wichtigen Beitrag, den immer noch viel zu sehr auf individuelle Glaubensfragen bezogenen Religionsunterricht gegenüber globalen und ökologischen Gerechtigkeitsfragen zu öffnen und diese ins Zentrum zu rücken. Er ermutigt, sich auch politischen und wirtschaftlichen Fragen und der Problematik unseres Wirtschaftssystems („Diese Wirtschaft tötet“, Papst Franziskus) im Religionsunterricht zu stellen und im Dreischritt „Erkennen - Bewerten - Handeln“ anzuregen, aktiv zu werden für eine nachhaltige und gerechte Welt. Der Aufsatz von Andreas Benk ist allen Religionslehrer*innen und allen Lehrenden in Theologie und Religionspädagogik zur dringenden Lektüre empfohlen! 